\title{
Força de preensão, capacidade funcional e qualidade de vida de indivíduos com câncer
}

\author{
Gripping strength, functional capacity and quality of life of individuals with cancer
}

Fuerza de agarre, capacidad funcional y calidad de vida de personas con cáncer

\author{
Anne Caroline Fonseca Duarte', Barbara Alice Silva², Patrick Roberto Avelino ${ }^{3}$ \\ Kênia Kiefer Parreiras de Menezes ${ }^{4}$
}

\begin{abstract}
RESUMO | O objetivo deste estudo foi avaliar a força, capacidade funcional e qualidade de vida de indivíduos com câncer em comparação com valores preditos em indivíduos saudáveis, bem como determinar se força e capacidade funcional são capazes de predizer a qualidade de vida dessa população. Foram avaliados 50 indivíduos com câncer, de acordo com força (dinamômetro Crown), capacidade funcional (teste de AVD-Glittre) e qualidade de vida (SF-36). Os resultados foram comparados aos valores de referência preditos em indivíduos saudáveis, pareados por idade e sexo, pelo teste two-sample Kolmogorov-Smirnov. O coeficiente de correlação de Spearman foi utilizado para avaliar as relações entre as variáveis, e a regressão linear múltipla foi utilizada para avaliar se força e capacidade funcional são capazes de predizer a qualidade de vida. Houve diferença significativa $(p<0,01)$ para força, capacidade funcional e qualidade de vida entre indivíduos com câncer e o predito em indivíduos saudáveis. Bem como, uma correlação significativa entre força e capacidade funcional $(\rho=-$ $0,58 ; p<0,01)$, entre força e qualidade de vida $(\rho=0,46$; $p<0,01$ ), e qualidade de vida e capacidade funcional $(\rho=-$ 0,$51 ; p<0,01$ ). A análise de regressão demonstrou que a capacidade funcional é preditora da qualidade de vida, sendo capaz, sozinha, de explicar 20\% $\left(R^{2}=0,195\right)$ dessa variável. Assim, indivíduos com câncer apresentaram diminuição significativa na força, capacidade funcional e qualidade de vida se comparados ao predito. Além disso,
\end{abstract}

todas essas variáveis estão correlacionadas em indivíduos com câncer, principalmente, a capacidade funcional e a qualidade de vida.

Descritores | Neoplasia, Força Muscular; Exercício; Qualidade de Vida.

ABSTRACT | This study aims to evaluate strength, functional capacity, and quality of life in individuals with cancer, compared with predicted values of healthy individuals, as well as to determine whether strength and functional capacity predict quality of life in this population. Fifty individuals with cancer were evaluated according to strength (dynamometer Crown), functional capacity (Glittre ADL test) and quality of life (SF-36). Results were compared with predicted reference values for healthy individuals, matched for age and gender, by the two-sample Kolmogorov-Smirnov test. Spearman's correlation coefficient was used to evaluate the correlations between variables. Multiple linear regression was used to investigate whether strength and functional capacity predict quality of life. There is significant difference $(p<0.01)$ for strength, functional capacity, and quality of life, between individuals with cancer and the predicted in healthy individuals. There was a significant correlation between strength and functional capacity $(\rho=-0.58 ; p<0.01)$, strength and quality of life $(\rho=0.46 ; p<0.01)$, and quality of life and functional capacity $(\rho=-0.51 ; p<0.01)$. Regression analysis showed that functional capacity is a predictor of quality of life and, alone, can explain $20 \%\left(R^{2}=0.195\right)$ of this

Trabalho realizado na Fundação Comunitária de Ensino Superior de Itabira - Itabira (MG), Brasil.

'Fundação Comunitária de Ensino Superior de Itabira - Itabira (MG), Brasil. E-mail: annecfduarte123@gmail.com. Orcid: 0000-0001-9722-9781

2Fundação Comunitária de Ensino Superior de Itabira - Itabira (MG), Brasil. E-mail: barbara.alice.binha@hotmail.com. Orcid: 0000-0001-7177-7958

${ }^{3}$ Faculdade de Santa Luzia - Itabira (MG), Brasil. E-mail: patrickpk4@yahoo.com.br. Orcid: 0000-0002-7248-4767 ${ }^{4}$ Fundação Comunitária de Ensino Superior de Itabira - Itabira (MG), Brasil. E-mail: keniakiefer@yahoo.com.br. Orcid: 0000-0002-9906-9555 
variable. Thus, individuals with cancer showed significant decrease in strength, functional capacity, and quality of life, when compared to predicted values. Moreover, all these variables are correlated, mainly functional capacity and quality of life.

Keywords | Neoplasms; Hand Strength; Exercise; Quality of Life.

RESUMEN | El objetivo de este estudio fue evaluar la fuerza, la capacidad funcional y la calidad de vida de los individuos con cáncer en comparación con los valores pronosticados en individuos sanos, así como determinar si la fuerza y la capacidad funcional son capaces de predecir la calidad de vida de esta población. Se evaluaron a cincuenta individuos con cáncer según la fuerza (dinamómetro Crown), la capacidad funcional (prueba de AVD-Glitro) y la calidad de vida (SF-36). Los resultados se compararon con los valores de referencia predichos en individuos sanos, pareados por edad y sexo, por la prueba two-sample Kolmogorov-Smirnov. Se utilizó el coeficiente de correlación de Spearman para evaluar las relaciones entre las variables, y la regresión lineal múltiple para evaluar si la fuerza y la capacidad funcional son capaces de predecir la calidad de vida. Hubo una diferencia significativa $(p<0,01)$ para la fuerza, la capacidad funcional y la calidad de vida entre los individuos con cáncer y la predicha en los individuos sanos. Además de una correlación significativa entre fuerza y capacidad funcional $(\rho=-0,58$; $p<0,01)$, fuerza y calidad de vida $(\rho=0,46 ; p<0,01)$, y calidad de vida y capacidad funcional $(\rho=-0,51 ; p<0,01)$. El análisis de regresión mostró que la capacidad funcional es predictora de la calidad de vida, pudiendo explicar, por sí sola, el 20\% $\left(R^{2}=0,195\right)$ de esta variable. Así los individuos con cáncer tuvieron una disminución significativa en la fuerza, la capacidad funcional y la calidad de vida en comparación con lo predicho. Además, todas estas variables están correlacionadas con individuos con cáncer, sobre todo la capacidad funcional y la calidad de vida.

Palabras clave | Neoplasia, Fuerza Muscular; Ejercicio; Calidad de vida.

\section{INTRODUÇÃO}

O câncer tem como características principais o crescimento e a proliferação de células irregulares, que se multiplicam até formar um aglomerado intitulado de tumor ${ }^{1}$. Quando maligno, o tumor impossibilita as atividades do organismo e altera o caminho do alimento e suprimento sanguíneo das células regulares ${ }^{1}$. O seu crescimento não é controlado e pode levar à morte do indivíduo ${ }^{2}$. As causas não são totalmente entendidas, porém existem fatores que podem elevar a sua aparição, como, por exemplo, obesidade e tabagismo ${ }^{2}$. Segundo a Organização Mundial da Saúde (OMS), em 2018, o câncer foi a segunda maior causa de morte no mundo, responsável por cerca de 9,6 milhões de mortes, perdendo apenas para doenças cardiovasculares ${ }^{3}$.

Os tratamentos mais comuns do câncer são cirurgia, radioterapia e terapia sistêmica ${ }^{4}$. Dentro da terapia sistêmica, a quimioterapia e imunoterapia são as mais conhecidas ${ }^{4}$. A quimioterapia consiste no uso de drogas que atuam em nível celular, prejudicando o crescimento e multiplicação das células cancerígenas ${ }^{5}$. No entanto, tais agentes quimioterápicos não apresentam preferência apenas pelas células tumorais, sendo tóxicos a todos os tecidos de rápida proliferação do corpo humano e, desse modo, afeta o organismo do indivíduo de forma geral ${ }^{5}$. Dentre as complicações enfrentadas pelos indivíduos com câncer, em decorrência do tratamento, destaca-se a fraqueza muscular ${ }^{6}$. As aparentes causas para essa condição seriam o aumento da taxa de gasto energético, aumento do processo de degradação de proteína por enzimas associado à diminuição da síntese proteica, e aumento da lipólise ${ }^{7}$. A fraqueza muscular nessa população pode resultar em internações mais longas e menor sobrevida, além de capacidade funcional reduzida e pior qualidade de vida ${ }^{8}$.

O termo "capacidade" descreve a aptidão de um indivíduo para executar uma tarefa ou uma ação em ambiente padronizado e, portanto, o constructo capacidade funcional visa indicar o nível máximo provável de funcionalidade que a pessoa é capaz de atingir em um dado domínio em um dado momento 9 . Por outro lado, a qualidade de vida pode ser definida como a percepção que o indivíduo tem sobre sua própria condição de vida, dentro do próprio contexto de cultura e sistema de valores, considerando seus objetivos de vida, expectativas, preocupações, limitações e restrições ${ }^{10}$. A função física restrita, em conjunto com os outros sintomas encontrados nessa população, como dor, enjoos e vômitos, depressão, dentre outros ${ }^{11,12}$, podem levar a um comprometimento da capacidade funcional e da qualidade de vida de indivíduos com câncer, assim como gerar um círculo vicioso ${ }^{13,14}$.

Dessa forma, estabelecer a magnitude das perdas de força, capacidade funcional e qualidade de vida de indivíduos com câncer, quando comparados com indivíduos saudáveis, além de definir uma possível relação entre essas variáveis, é fundamental para a reabilitação dessas pessoas. Nesse contexto, estudos prévios objetivaram investigar a força 
muscular de indivíduos com câncer, comparando-os com indivíduos saudáveis ${ }^{15-17}$. No entanto, enquanto Harrington et al. ${ }^{15} \mathrm{e}$ Niederer et al. ${ }^{17}$ encontraram diferença significativa na força dos membros superiores e de quadríceps de indivíduos com câncer em relação aos indivíduos saudáveis, outro estudo não encontrou diferença significativa na força manual e de extensores de joelho nessa população, quando comparados aos saudáveis ${ }^{16}$. Tais resultados contraditórios reafirmam a necessidade de mais estudos sobre o assunto. Em relação à capacidade funcional e qualidade de vida, não foram encontrados estudos que tenham comparado indivíduos com câncer com indivíduos saudáveis, ou mesmo com valores preditos.

O objetivo deste estudo foi avaliar a força, capacidade funcional e qualidade de vida de indivíduos com câncer em comparação com valores preditos em indivíduos saudáveis, além de investigar possíveis correlações entre essas variáveis, e determinar se força e capacidade funcional são capazes de predizer a qualidade de vida dessa população.

\section{METODOLOGIA}

Trata-se de um estudo observacional transversal, realizado na Fundação Comunitária de Ensino Superior de Itabira (Funcesi).

\section{Participantes}

Os participantes foram recrutados no Centro Oncológico Itabira (COI) e na comunidade em geral, entre os meses de agosto a outubro de 2019. Para as coletas no COI, o coordenador assinou uma carta de anuência, autorizando a realização do estudo com os pacientes do centro. Para a inclusão no estudo, os participantes obedeceram aos seguintes critérios: (1) indivíduos de ambos os sexos, sem restrição de idade; (2) indivíduos diagnosticados com câncer, independentemente do tempo de diagnóstico ou local de acometimento; (3) ser capaz de realizar todos os testes. Foram excluídos os participantes que indicaram ou apresentaram situações físicas, funcionais e/ou mentais que poderiam impossibilitar ou interferir nos resultados dos testes. Todos os indivíduos incluídos no estudo assinaram o termo de consentimento livre e esclarecido (TCLE).

\section{Procedimentos}

Inicialmente, foram coletados dados demográficos e clínicos dos participantes, a fim de caracterizar a amostra. Posteriormente, após análise dos critérios de inclusão e assinatura do TCLE, foram recolhidas as seguintes medidas de desfecho: força de preensão manual, capacidade funcional e qualidade de vida. Todas as coletas foram realizadas no auditório do Hospital Nossa Senhora das Dores e na Clínica Escola da Funcesi.

\section{Força de preensão}

A força de preensão foi avaliada pelo dinamômetro manual (Crown, com capacidade de 50kgf) que, segundo estudos prévios, pode ser utilizado como indicador da força global do indivíduo ${ }^{18}$. Durante a coleta, o indivíduo permaneceu sentado em uma cadeira sem descanso para os braços, com a coluna ereta, joelhos mantidos em flexão de $90^{\circ}$, ombros com posicionamento de adução e rotação neutra, com flexão de cotovelo no ângulo de $90^{\circ}$, antebraço em neutro, e punho com uma leve extensão de $30^{\circ 19}$. A avaliação foi realizada de forma alternada entre a mão dominante e não dominante, com três aferições para cada lado $^{19}$. O maior valor encontrado foi registrado para cada indivíduo. Os resultados foram comparados aos valores de referência preditos em indivíduos saudáveis, pareados por idade e sexo ${ }^{20}$.

\section{Capacidade funcional}

A capacidade funcional foi avaliada através do teste de AVD-Glittre (TGlittre). Nesse teste, partindo da posição sentada, os indivíduos são instruídos a se levantar e andar por um corredor de 10 metros, passando por uma escada, que se encontra na metade desse corredor, até chegar a uma estante. As prateleiras da estante ficam posicionadas na altura da cintura escapular e da cintura pélvica. $\mathrm{O}$ sujeito deve mover três pesos, de um quilograma $(\mathrm{kg})$ cada, a partir da prateleira superior para a prateleira mais baixa e, depois, para o chão. A seguir, deve realizar a sequência inversa, de modo que cada peso deve ser colocado novamente na prateleira superior. Então, o indivíduo retorna até se assentar na cadeira e reinicia o percurso. $\mathrm{O}$ teste termina quando o indivíduo completa cinco percursos completos. É permitido que ele descanse durante o teste, entretanto, é orientado a retornar o mais rápido possível. Não é dado nenhum incentivo durante o percurso, e os indivíduos devem usar uma mochila contendo um peso de $2,5 \mathrm{~kg}$ (mulheres) ou $5 \mathrm{~kg}$ (homens). A variável desfecho é o tempo gasto para finalizar o teste $^{21-23}$. Foram realizadas medições da pressão arterial, frequência cardíaca, saturação de oxigênio e fadiga com o indivíduo em repouso inicial, ao final, e cinco minutos após o término. Os valores encontrados nos indivíduos 
com câncer foram comparados aos preditos em indivíduos saudáveis ${ }^{24}$.

\section{Qualidade de vida}

A qualidade de vida foi avaliada por meio do questionário genérico Short-Form Health Survey (SF-36), que abrange oito domínios: capacidade funcional, limitação física, dor, estado geral de saúde, vitalidade, aspectos sociais, aspectos emocionais e saúde mental ${ }^{25}$. O SF-36 é aplicado em forma de entrevista e sua pontuação varia de 0 a 100, sendo que quanto maior o valor encontrado, melhor a qualidade de vida do indivíduo. Os valores identificados nos indivíduos com câncer também foram comparados ao preditos em indivíduos saudáveis, pareados por idade e $\operatorname{sexo}^{26}$.

\section{Cálculo amostral}

O cálculo amostral foi baseado nos coeficientes de correlação entre as variáveis, encontrados em um estudo piloto com 10 indivíduos. Considerando um alfa de 0,05 , uma potência de 0,90 e assumindo o menor coeficiente encontrado no piloto $(\rho=0,40)$, entre capacidade funcional e qualidade de vida, a amostra necessária seria de 50 indivíduos ${ }^{27}$. Esse tamanho de amostra também é suficiente para a análise de regressão, considerando a inserção de duas variáveis independentes (força de preensão e capacidade funcional), uma vez que a fórmula utilizada para o cálculo seria $n=30+10 k$, onde " $k$ " é o número de possíveis variáveis preditoras a serem inclusas ${ }^{28}$.

\section{Análise estatística}

Estatísticas descritivas e testes de normalidade (Kolmogorov-Smirnov) foram realizados para todas as variáveis. Foi utilizado o teste two-sample KolmogorovSmirnov para avaliação da diferença entre os valores encontrados em indivíduos com câncer e os valores preditos em indivíduos saudáveis. $\mathrm{O}$ coeficiente de correlação de Spearman foi empregado para analisar as correlações entre força de preensão, capacidade funcional e qualidade de vida. A força das correlações foi classificada como baixa $(\rho<0,30)$, moderada $(0,30 \leq \rho \leq 0,50)$ e alta $(\rho>0,50)^{29}$. Regressão linear múltipla, método steprwise, foi usada para avaliar qual(is) variável(eis) é (são) capaz(es) de predizer a qualidade de vida dessa população. Todas as análises foram realizadas com o software estatístico SPSS 23.0, com uma significância de 5\%.

\section{RESULTADOS}

\section{Características dos participantes}

Dos 161 indivíduos convidados para participar do estudo, 95 recusaram ou não se encaixaram nos critérios de inclusão, e 16 não compareceram à avaliação agendada, por motivos de dificuldade de deslocamento. Assim, a amostra total foi composta por 50 participantes (Figura 1), sendo 19 (38\%) homens. A idade média dos participantes foi de 59 anos e desvio-padrão (DP) 12. Os tipos de câncer encontrados foram 11 , no total, sendo os de maior prevalência o de mama 26 (52\%) e próstata 9 (18\%), seguidos dos de reto 4 (8\%), estômago 2 (4\%) e pâncreas 2 (4\%). Os tratamentos que os indivíduos estavam submetidos eram: quimioterapia 40 (80\%), hormonioterapia 7 (14\%) e radioterapia 3 (6\%). O tempo médio desde o diagnóstico do câncer foi de 21 meses (DP 19). As características gerais dos participantes encontram-se na Tabela 1.

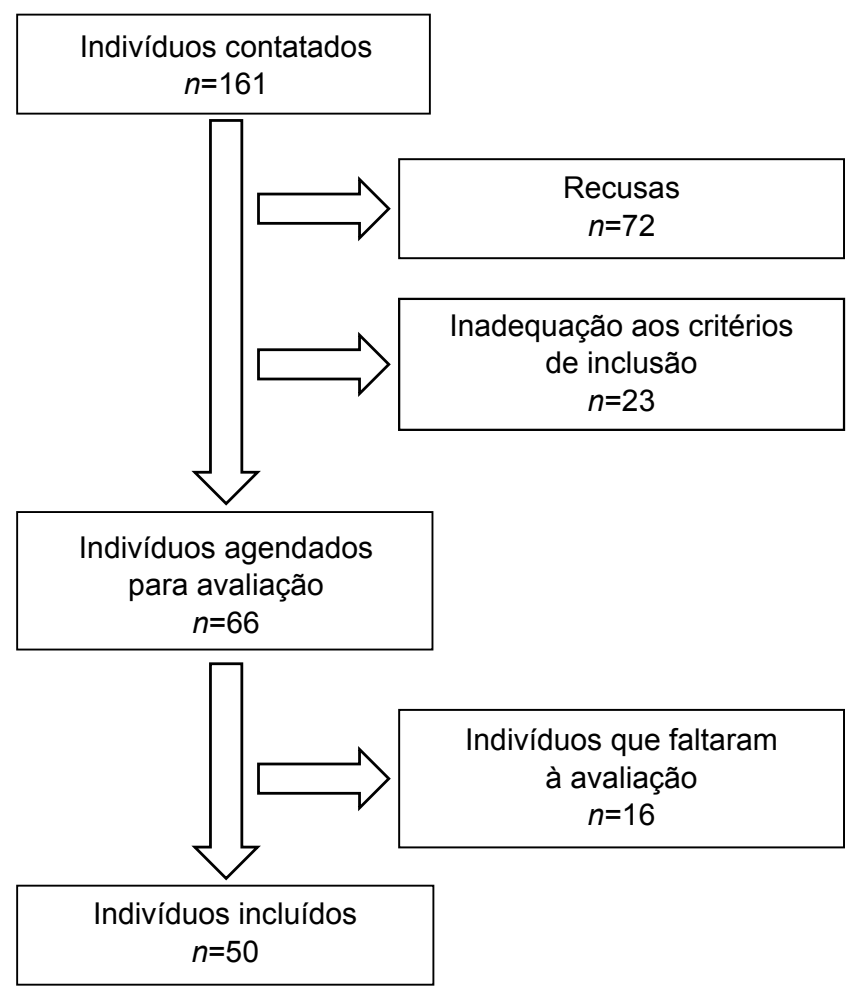

Figura 1. Fluxograma de inclusão dos participantes $(n=50)$

Tabela 1. Características dos participantes $(n=50)$ 


$\begin{array}{lc}\text { Tabela 1. Continuação } & \\ \text { Localização do câncer (\%) } & 26(52) \\ \text { Mama } & 9(18) \\ \text { Próstata } & 2(4) \\ \text { Estômago } & 2(4) \\ \text { Pâncreas } & 4(8) \\ \text { Reto } & 1(2) \\ \text { Esôfago } & 1(2) \\ \text { Fígado } & 1(2) \\ \text { Pulmão } & 1(2) \\ \text { Intestino } & 1(2) \\ \text { Útero } & 1(2) \\ \text { Cérebro } & 1(2) \\ \text { Ovário } & \\ \text { Tratamento (\%) } & \\ \text { Quimioterapia } & 40(80) \\ \text { Hormonioterapia } & 7(14) \\ \text { Radioterapia } & 3(6) \\ \text { Tempo de diagnóstico (meses), média (DP) } & 21(19) \\ \text { DP. desvio-padrão } & \end{array}$

\section{Diferença entre os valores encontrados em indivíduos com câncer e os valores preditos em indivíduos saudáveis}

Foi encontrada diferença significativa $(p<0,001)$ entre a força de preensão manual dos indivíduos com câncer (média 25, DP 9) e os valores preditos em indivíduos saudáveis (média 29, DP 8). Também foi identificada diferença significativa $(p<0,01)$ quanto à capacidade funcional entre os valores da amostra (média 264, DP 85 ) e do predito (média 255,DP 61).Por fim, de forma similar, a qualidade de vida média dos indivíduos com câncer (média 56,13, DP 23,38$)$ apresentou diferença significativa $(p<0,001)$ quando comparada ao valor predito (média $73,10, \mathrm{DP}$ $25,16)$. Os resultados encontram-se na Tabela 2.

Tabela 2. Diferença da força de preensão, capacidade funcional e qualidade de vida entre os valores encontrados em indivíduos com câncer e os valores preditos em indivíduos saudáveis

$\begin{array}{lccc} & \begin{array}{l}\text { Indivíduos } \\ \text { com câncer }\end{array} & \begin{array}{c}\text { Indivíduo } \\ \text { saudável } \\ \text { (Predito) }\end{array} & \begin{array}{c}\text { Two-sample } \\ \text { Kolmogorov- } \\ \text { Smirnov test }\end{array} \\ \begin{array}{l}\text { Força de preensão } \\ \text { (Dinamômetro - kgf) }\end{array} & 25(9) & 29(8) & \mathrm{p}<0,001 \\ \begin{array}{l}\text { Capacidade funcional } \\ \text { (AVD Glittre - segundos) }\end{array} & 264(85) & 255(61) & \mathrm{p}<0,01 \\ \begin{array}{l}\text { Qualidade de vida } \\ \text { (SF-36 - 0-100 pontos) }\end{array} & 56,13 & 73,10 & \mathrm{p}<0,001\end{array}$

SF-36: Short-Form Health Survey.

\section{Correlação e análise de regressão entre força de preensão, capacidade funcional e qualidade de vida em indivíduos com câncer}

Foi descoberta correlação significativa, de magnitude alta, entre força e capacidade funcional $(\rho=-0,58 ; p<0,01)$, e moderada entre força e qualidade de vida $(\rho=0,46$; $p<0,01)$. Além disso, também foi encontrada correlação significativa, de magnitude alta, entre capacidade funcional e qualidade de vida $(\rho=-0,51 ; p<0,01)$. Os valores de cada uma das correlações encontram-se na Tabela 3. $\mathrm{Na}$ análise de regressão, apenas a capacidade funcional permaneceu no modelo $(\mathrm{p}<0,01)$ como preditora da qualidade de vida, sendo capaz, sozinha, de explicar $20 \%\left(R^{2}=0,195\right)$ dessa variável.

Tabela 3. Coeficiente de correlação de Spearman entre força de preensão, capacidade funcional e qualidade de vida

$\begin{array}{lccc} & \begin{array}{c}\text { Força de } \\ \text { preensão }\end{array} & \begin{array}{c}\text { Capacidade } \\ \text { funcional }\end{array} & \begin{array}{c}\text { Qualidade } \\ \text { de vida }\end{array} \\ \begin{array}{l}\text { Força de preensão } \\ \text { (Dinamômetro - kgf) }\end{array} & - & \begin{array}{c}\rho=-0,58 \\ (p<0,01)\end{array} & \rho=0,46 \\ & & & (p<0,01) \\ \text { Capacidade funcional } & - & - & \rho=-0,51 \\ \text { (AVD Glittre - segundos) } & - & & (p<0,01) \\ \begin{array}{l}\text { Qualidade de vida } \\ \text { (SF-36-0-100) }\end{array} & - & - & -\end{array}$

SF-36: Short-Form Health Survey.

\section{DISCUSSÃO}

Este estudo teve como finalidade avaliar a força, capacidade funcional e qualidade de vida de indivíduos com câncer, quando comparados com valores preditos em indivíduos saudáveis, além de investigar possíveis correlações entre as variáveis e a melhor preditora para qualidade de vida. Os resultados demonstraram diferença significativa entre todas as variáveis coletadas e seus valores preditos, indicando que indivíduos com câncer apresentam força, capacidade funcional e qualidade de vida inferior aos valores preditos em indivíduos saudáveis. Bem como, houve correlação significativa entre todas as variáveis, demonstrando que os indivíduos com redução da força de preensão apresentam diminuição na capacidade funcional e menor percepção da qualidade de vida. Por fim, a capacidade funcional pode ser considerada preditora da qualidade de vida dessa população.

A força média encontrada nos participantes avaliados $(25 \mathrm{~kg})$ foi significativamente menor, quando comparada aos valores preditos $(29 \mathrm{~kg})$. Pesquisas recentes na literatura corroboram os resultados deste estudo, 
reportando diminuição da força nas amostras analisadas de indivíduos com câncer em relação aos saudáveis ${ }^{15,17}$. De fato, indivíduos com câncer sofrem modificações devido à própria doença e ao tratamento administrado ${ }^{30}$. Essas alterações em conjunto podem estar adjuntas à depressão e perda de apetite, que iniciam um ciclo vicioso, levando à perda de massa muscular e queda nas atividades físicas, que resultam em uma fraqueza global ${ }^{4}$. Um estudo recente, com 51 indivíduos com câncer, embora tenha encontrado um valor de força de preensão manual médio similar ao presente estudo $(23 \mathrm{~kg})$, não identificou diferença significativa ao comparar com indivíduos saudáveis ${ }^{31}$. No entanto, vale ressaltar que Silva et al. ${ }^{31}$ utilizaram como grupo-controle idosos com diferentes condições musculoesqueléticas, o que pode ter comprometido seus resultados.

Este estudo também demonstrou uma diminuição na capacidade funcional de indivíduos com câncer. Tais resultados eram esperados, uma vez que a redução na capacidade funcional está ligada a uma maior dependência para realização de atividades diárias ${ }^{32}$. Essa redução ocorre devido à possível alteração, pelo tratamento e pela doença, dos sistemas cognitivo, locomotor e de comunicação, fundamentais para a execução de tarefas cotidianas ${ }^{32}$. $O$ resultado desta pesquisa se assemelha a achados de um estudo prévio ${ }^{13}$, que avaliou a capacidade funcional de 128 mulheres, e reportou redução significativa da capacidade funcional nessas pacientes. No entanto, tais dados não foram comparados com indivíduos saudáveis ou valores preditos ${ }^{13}$. Um outro estudo também reportou uma capacidade funcional significativamente reduzida em indivíduos com câncer, quando comparados aos saudáveis ${ }^{17}$. Porém, tal estudo avaliou a capacidade funcional pelo equilíbrio, velocidade de marcha e força de quadríceps ${ }^{17}$, medidas essas que não podem ser consideradas para avaliação deste constructo.

Em relação à qualidade de vida, pesquisas anteriores avaliaram esse domínio em indivíduos com câncer e reportaram, similar ao presente estudo, valores reduzidos ${ }^{33,34}$. No entanto, tal conclusão foi baseada apenas na interpretação dos autores, visto que os valores encontrados não foram comparados com os de indivíduos saudáveis ou preditos. De fato, a capacidade de realizar atividades diárias, a satisfação e os níveis de funcionalidade são essenciais para determinar a qualidade de vida em sobreviventes de câncer de mama ${ }^{33}$. Sintomas persistentes associados aos efeitos adversos do tratamento, como dor e fadiga, podem levar a uma capacidade funcional reduzida, como demonstrado previamente, afetando diretamente a percepção da qualidade de vida desses indivíduos, que se sentem mais dependentes, deprimidos, e menos satisfeitos com sua condição atual de vida ${ }^{30}$.

Por fim, este estudo identificou correlação entre todas as variáveis avaliadas. Dessa forma, indivíduos com câncer que apresentam diminuição na força de preensão necessitam de mais tempo para realizar atividades cotidianas e, assim, apresentam pior percepção da sua qualidade de vida. Resultados similares foram encontrados em estudos prévios, que também analisaram a correlação entre essas variáveis e descobriram resultados significativos entre força e qualidade de vida ${ }^{35}$, e capacidade funcional e qualidade de $\operatorname{vida}^{36}$. Embora seja sugestivo que a fraqueza muscular desses indivíduos pode ocasionar uma diminuição da capacidade funcional e consequente piora da qualidade de vida, tal interpretação merece cautela ${ }^{37}$. Uma análise de correlação não tem potencial de determinar uma relação de causa e efeito, portanto não podemos afirmar que uma condição causa a outra, mas, apenas, que estão relacionadas ${ }^{27}$. Por outro lado, a regressão fornece o coeficiente de determinação ou de explicação $\left(\mathrm{R}^{2}\right)$, que indica quantos por cento a variação explicada pela regressão representa sobre a variação tota ${ }^{27}$. No presente estudo, ainda que força e capacidade funcional estejam correlacionadas com qualidade de vida, somente a segunda foi capaz de predizer o escore do SF-36, indicando que, quando ocorrem mudanças na qualidade de vida, 20\% dessa alteração pode ser explicada por modificações na capacidade funcional do indivíduo com câncer.

O principal ponto positivo deste estudo é a realização de uma pesquisa, que preenche uma importante lacuna da literatura, com a utilização de instrumentos de baixo custo, fácil manuseio, rápida aplicação, com propriedades de medidas adequadas, e que podem ser usados em ambientes laborais, escolares e hospitalares. No entanto, podemos citar como principal limitação do estudo a utilização de valores preditos encontrados na literatura, que podem não ser tão sensíveis quanto àqueles diretamente coletados de uma amostra de indivíduos saudáveis. Além disso, o valor de referência da capacidade funcional foi baseado em um estudo indiano, uma vez que não possuem valores brasileiros publicados, o que talvez não seja generalizável para a nossa população.

\section{CONCLUSÃO}

Os resultados deste estudo demonstraram que indivíduos com câncer apresentam redução da força e 
capacidade funcional, assim como piora da qualidade de vida significativas, quando comparadas aos valores preditos em indivíduos saudáveis. Além disso, existe uma correlação entre todas essas variáveis, indicando que os indivíduos com redução da força de preensão apresentam diminuição na capacidade funcional e uma menor percepção da qualidade de vida, sendo que $20 \%$ desta última pode ser explicada apenas pela capacidade funcional. Tais resultados são clinicamente relevantes, pois demonstram a importância da força e, principalmente a capacidade funcional desses indivíduos, para a melhor tomada de decisão clínica, visando uma melhor qualidade de vida.

\section{REFERÊNCIAS}

1. Feitelson MA, Arzumanyan A, Kulathinal RJ, Blain SW, Holcombe RF, Mahajna J, et al. Sustained proliferation in cancer: mechanisms and novel therapeutic targets. Semin Cancer Biol. 2015;35(Suppl):S25-S54. doi: 10.1016/j.semcancer.2015.02.006

2. Casás-Selves M, DeGregori J. How cancer shapes evolution, and how evolution shapes cancer. Evolution (N Y). 2011;4(4):62434. doi: 10.1007/s12052-011-0373-y

3. Siegel RL, Miller KD, Jemal A. Cancer statistics, 2019. CA Cancer J Clin. 2019;69(1):7-34. doi: 10.3322/caac.21551

4. Courneya KS. Exercise in cancer survivors: an overview of research. Med Sci Sports Exerc. 2003;35(11):1846-52. doi: 10.1249/01.mss.0000093622.41587.b6

5. Li X, Hou Y, Zhao J, Zhao J, Wang S, Fang J. Combination of chemotherapy and oxidative stress to enhance cancer cell apoptosis. Chem Sci. 2020;11:3215-22. doi: 10.1039/C9SC05997K

6. Milgrom DP, Lad NL, Koniaris LG, Zimmers TA. Bone pain and muscle weakness in cancer patients. Curr Osteoporos Rep. 2017;15(2):76-87. doi: 10.1007/s11914-017-0354-3

7. Mendes MCS, Pimentel GD, Costa FO, Carvalheira JBC. Molecular and neuroendocrine mechanisms of cancer cachexia. J Endocrinol. 2015;226(3):R29-43. doi: 10.1530/JOE-15-0170

8. Sjøblom B, Grønberg BH, Wentzel-Larsen T, Baracos VE, Hjermstad MJ, Aass N, et al. Skeletal muscle radiodensity is prognostic for survival in patients with advanced non-small cell lung cancer. Clin Nutr. 2016;35(6):1386-93. doi: 10.1016/j. clnu.2016.03.010

9. Organização Mundial da Saúde. Classificação internacional de funcionalidade, incapacidade e saúde. Lisboa: Direcção-Geral da Saúde; 2004.

10. Cieza A, Stucki G. The International Classification of Functioning Disability and Health: its development process and content validity. Eur J Phys Rehabil Med [Internet]. 2008 [cited 2021 Feb 20];44(3):303-13. Available from: https://www. minervamedica.it/en/journals/europa-medicophysica/article. php?cod=R33Y2008N03A0303

11. Irwin KE, Greer JA, Khatib J, Temel JS, Pirl WF. Early palliative care and metastatic non-small cell lung cancer: potential mechanisms of prolonged survival. Chron Respir Dis. 2013;10(1):35-47. doi: 10.1177/1479972312471549

12. Zhu XC, Zhang JL, Ge CT, Yu YY, Wang P, Yuan TF, et al. Advances in cancer pain from bone metastasis. Drug Des Devel Ther. 2015:9:4239-45. doi: 10.2147/DDDT.S87568

13. Kokkonen K, Saarto T, Mäkinen T, Pohjola L, Kautio H, Järvenpää $\mathrm{S}$, et al. The functional capacity and quality of life of women with advanced breast cancer. Breast Cancer. 2017;24(1):128-36. doi: 10.1007/s12282-016-0687-2

14. Salvetti MG, Machado CSP, Donato SCT, Silva AM. Prevalence of symptoms and quality of life of cancer patients. Rev Bras Enferm. 2020;73(2):e20180287. doi: 10.1590/0034-7167-2018-0287

15. Harrington S, Padua D, Battaglini C, Michener LA, Giuliani C, Myers J, et al. Comparison of shoulder flexibility, strength, and function between breast cancer survivors and healthy participants. J Cancer Surviv. 2011;5(2):167-74. doi: 10.1007/ s11764-010-0168-0

16. Morishita S, Tsubaki A, Fu JB, Mitobe Y, Onishi H, Tsuji T. Cancer survivors exhibit a different relationship between muscle strength and health-related quality of life/fatigue compared to healthy subjects. Eur J Cancer Care. 2018;27(4):e12856. doi: 10.1111/ecc.12856

17. Niederer D, Schmidt K, Vogt L, Egen J, Klingler J, Hübscher M, et al. Functional capacity and fear of falling in cancer patients undergoing chemotherapy. Gait Posture. 2014;39(3):865-9. doi: 10.1016/j.gaitpost.2013.11.014

18. Porto JM, Nakaishi APM, Cangussu-Oliveira LM, Freire RC Jr, Spilla SB, Abreu DCC. Relationship between grip strength and global muscle strength in community-dwelling older people. Arch Gerontol Geriatr. 2019;82:273-278. doi: 10.1016/j. archger.2019.03.005

19. Musalek C, Kirchengast S. Grip Strength as an Indicator of Health-Related Quality of Life in Old Age-A Pilot Study. Int J Environ Res Public Health. 2017;14(12):1447. doi: 10.3390/ ijerph14121447

20. Novaes RD, Miranda AS, Silva JO, Tavares BVF, Dourado VZ. Equações de referência para a predição da força de preensão manual em brasileiros de meia idade e idosos. Fisioter Pesqui. 2009;16(3):217-22. doi: 10.1590/S1809-29502009000300005

21. Skumlien S, Hagelund T, Bjørtuft O, Ryg MS. A field test of functional status as performance of activities of daily living in COPD patients. Respir Med. 2006;100(2):316-23. doi: 10.1016/j. rmed.2005.04.022

22. José A, Dal Corso S. Reproducibility of the six-minute walk test and Glittre ADL-test in patients hospitalized for acute and exacerbated chronic lung disease. Braz J Phys Ther. 2015;19(3):235-42. doi: 10.1590/bjpt-rbf.2014.0092

23. Araujo CL, Gulart A, Munari A, Zanotto J, Schneider B, Dal Lago P, et al. Reproducibility and learning effect of the Glittre ADL-test. Eur Resp J. 2019;54:PA1208. doi: 10.1183/13993003. congress-2019.PA1208

24. Paul F, Alaparthi GK, Krishnan S, Ramakrishna A, Acharya V, Acharya P. Reference values for glittre activities of the daily living test in healthy subjects among Indian population: a cross-sectional study. Curr Respir Med Rev. 2019;15(1):15-20. doi: 10.2174/1573398X15666190112145934 
25. Finkelstein FO, van Nooten F, Wiklund I, Trundell D, Cella D. Measurement properties of the Short Form-36 (SF-36) and the Functional Assessment of Cancer Therapy - Anemia (FACT$A n$ ) in patients with anemia associated with chronic kidney disease. Health Qual Life Outcomes. 2018;16(1):111. doi: 10.1186/ s12955-018-0933-8

26. Cruz LN, Fleck MPA, Oliveira MR, Camey SA, Hoffmann JF, Bagattini AM, et al. Health-related quality of life in Brazil: normative data for the SF-36 in a general population sample in the south of the country. Ciênc Saúde Coletiva. 2013;18(7):191121. doi: 10.1590/S1413-81232013000700006

27. Portney LG, Watkins MP. Foundations of clinical research: applications to practice. 3rd ed. Upper Saddle River: Pearson; 2009.

28. Knapp TR, Campbell-Heider N. Numbers of observations and variables in multivariate analyses. West J Nurs Res. 1989;11(5):634-41. doi: 10.1177/019394598901100517

29. Cohen J. Statistical power analysis for the behavioral sciences. 2nd ed. Hillsdale: Lawrence Erlbaum Associates; 1988.

30. Nayak MG, George A, Vidyasagar MS, Mathew S, Nayak S, Nayak BS, et al. Quality of Life among Cancer Patients. Indian J Palliat Care. 2017;23(4):445-50. doi: 10.4103/IJPC.IJPC_82_17

31. Silva GA, Silva GD, Cabral AMBO, Santiago ERC, Araújo ERS, Rosa EPC, et al. Estado nutricional e capacidade funcional de idosos oncológicos hospitalizados. Nutr Clín Diet Hosp. 2019;39(2):5462. doi: 10.12873/392gabriela
32. Pereira EEB, Santos NB, Sarges ESNF. Avaliação da capacidade funcional do paciente oncogeriátrico hospitalizado. Rev Pan-Amaz Saude. 2014;5(4):37-44. doi: 10.5123/ S2176-62232014000400005

33. Santos LN, Castaneda L, Aguiar SS, Thuler LCS, Koifman RJ, Bergmann A. Health-related quality of life in women with cervical cancer. Rev Bras Ginecol Obstet. 2019;41(4):242-8. doi: 10.1055/s-0039-1683355

34. Mücke T, Koschinski J, Wolff KD, Kanatas A, Mitchell DA, Loeffelbein DJ, et al. Quality of life after different oncologic interventions in head and neck cancer patients. J Craniomaxillofac Surg. 2015;43(9):1895-8. doi: 10.1016/j. jcms.2015.08.005

35. Queiroz MSC, Wiegert EVM, Lima LC, Oliveira LC. Associação entre sarcopenia, estado nutricional e qualidade de vida em pacientes com câncer avançado em cuidados paliativos. Rev Bras Cancerol. 2018;64(1):69-75. doi: 10.32635/2176-9745. RBC.2018v64n1.120

36. Costa WA, Eleutério J Jr, Giraldo PC, Gonçalves AK. Quality of life in breast cancer survivors. Rev Assoc Med Bras. 2017;63(7):5839. doi: 10.1590/1806-9282.63.07.583

37. Frio CC, Pretto ADB, Gonzales MC, Pastore CA. Influência da composição corporal sobre a qualidade de vida de pacientes com câncer. Rev Bras Cancerol. 2015;61(4):351-7. doi: 10.32635/2176-9745.RBC.2015v61n4.224 